\title{
Missed diagnoses among elderly patients discharged from an accident and emergency department
}

\author{
S A Khan, F G Miskelly, J S Platt, B K Bhattachryya
}

\begin{abstract}
Objective-To investigate how often elderly patients are discharged from an accident and emergency (A\&E) department with unrecognised but remediable problems.

Methods-Over a period of six months, 174 elderly patients fulfilling inclusion criteria for the study were discharged from A\&E, and of these 97 (56\%) agreed to be reviewed in the day hospital. They were assessed by a doctor, nurse, physiotherapist, occupational therapist, speech therapist, and social worker. A full blood count, urea and electrolytes, liver and thyroid function tests, a chest radiograph, and an electrocardiogram were performed. A Barthel activity of daily living index was performed on the first visit and before discharge.

Results-28\% had missed diagnoses which benefited from day hospital attendance and $a$ further 13 patients had been admitted before they could attend day hospital. Those patients presenting with falls and living alone constituted a high risk group.

Conclusions-Elderly patients attending A\&E merit special consideration to detect underlying medical or social problems.

( $\mathcal{F}$ Accid Emerg Med 1996;13:256-257)
\end{abstract}

Key terms: accident and emergency; elderly; missed diagnosis; day hospital

We were concerned to notice that a proportion of elderly patients admitted to our hospital had recently been discharged from the accident and emergency (A\&E) department. There is evidence that remediable problems are missed in some elderly patients discharged from an $A \& E$ department ${ }^{1}$ and it has been suggested that these patients would benefit from a health visitor referral. ${ }^{2}$ The purpose of this study was to assess the extent to which remediable problems in elderly patients may be missed during $\mathrm{A} \& \mathrm{E}$ attendance and whether people of over 80 years of age merit special consideration.

\section{Methods}

This study was conducted at a large district general hospital in West London. The A\&E records of patients over 80 years attending the department in the previous 24 hours were collected daily. Those discharged were invited to attend the geriatric day hospital $(\mathrm{GDH})$ for further assessment. We excluded the following groups of patients:

(a) patients with arranged follow up in another hospital department;

(b) those living in nursing homes;

(c) those living outside the hospital's catchment area;

(d) those with severe dementia;

(e) those attending $A \& E$ for self limiting surgical or medical problems such as epistaxis, sprained wrist, dog bites, bee stings, or a change of urinary catheter.

Those failing to attend the GDH were given up to three appointments and if necessary contacted by telephone. The GDH attendance involved assessment by doctor, nurse, physiotherapist, occupational therapist, speech therapist, and social worker. A full blood count, urea and electrolytes, liver and thyroid function tests, a chest radiograph, and an electrocardiogram were performed. A Barthel activity of daily living index was performed on the first visit and before discharge.

Patients were classified as benefiting from GDH attendance if they showed improvement in their Barthel score, or had marked clinical improvement on medical treatment or paramedical input. Marked clinical improvement was defined as the discovery of an important treatable condition. Similarly patients with needs warranting hospital admission for successful treatment were classified as having benefited. In paramedical terms it was an improvement in mobility sufficient to allow the patient to continue living at home or the provision of additional services necessary for continued stay at home.

\section{Results}

Over a six month period, 377 patients above 80 years of age attended and were discharged from the $A \& E$ (table). Of these, 174 patients fulfilled the inclusion criteria and were invited to attend GDH for further assessment. From the 174 who were invited, 64 refused and 13 were admitted to hospital before their GDH visit.

Details of the patients

\begin{tabular}{lr}
\hline A\&E attenders & 377 \\
Invited to GDH & 174 \\
Attended GDH & 97 \\
Refused & 64 \\
Admitted before GDH visit & 13 \\
Benefited from GDH & 13 \\
Medical outpatient follow up & 7 \\
Admitted after GDH visit & 7
\end{tabular}

GDH, geriatric day hospital. 
Therefore 97 patients were assessed in the GDH of whom 68 were women and 29 men. Their age range was 80 to 99 years. Of these, $73 \%$ of the patients lived at home, $20 \%$ in residential homes, and $7 \%$ in warden controlled accommodation.

Among the 203 patients who were not invited to attend GDH, approximately $15 \%$ were attending other outpatient clinics already. The median interval between the patient's discharge from the $A \& E$ and visit to the GDH was 18 days.

Twenty seven $(28 \%)$ of the 97 patients benefited from their attendance at the GDH as follows: seven patients improved their Barthel score after $\mathrm{GDH}$ follow up; six benefited from intervention by either the social worker, speech therapist, or medical therapy; seven were invited for further follow up in the outpatient clinic for conditions such as anaemia, haematuria, hypertension, or postural hypotension; seven were admitted to the hospital from the GDH for further management of their problems, for example, Parkinson's disease, congestive cardiac failure, or fractured pelvis.

Therefore, 20 patients were admitted to hospital before or after their assessment in the GDH. All of these patients initially presented to $A \& E$ with falls and were later admitted to hospital with medical, surgical, or orthopaedic diagnoses, mostly fractures. Of these, 18 were living alone in the community. The diagnoses most frequently missed included immobility sufficient to preclude independent existence at home, dementia requiring intervention, anaemia, and poor social circumstances.

\section{Discussion}

Many papers have confirmed the large burden of problems in the elderly since the original description by Williamson et al in $1964 .^{3}$ Often the first point of contact of these patients with the medical establishment is the $A \& E$ department. Unfortunately, A\&E departments are usually very busy and focused upon treating the patient's immediate presenting complaint. This study was designed to assess whether the current operational policy of $A \& E$ department was adequate to detect problems in elderly patients not immediately related to the presenting complaint. Although this study was conducted only in one hospital we believe the results may be relevant to $A \& E$ departments in most district general hospitals.
It was not feasible to attempt to assess all patients who had attended $A \& E$, since some had obviously isolated reasons for attendance such as epistaxis, sprained wrist, or a change of urinary catheter. They would have been unlikely to agree to any further follow up or investigations. The patients we selected were those we expected to have a higher incidence of "unmet need". Even then 64 patients refused to attend; the reason stated in $91 \%$ of cases was that they were feeling well and did not see any need for a hospital visit. Although the level of disability and disease in the refusal group is not known, there is evidence to suggest that those who do not respond to health checks are no less disabled than those who do. ${ }^{4}$

These patients had all been discharged from the $A \& E$ without arranged follow up and yet a total of $28 \%$ of those attending were either admitted to hospital or benefited from intensive medical and paramedical intervention, and a further 13 had been admitted before they could attend GDH. It is possible that the patients' condition changed between the $A \& E$ and GDH attendances but this is unlikely due to the nature of the problems detected.

Interestingly $69 \%$ of the 174 patients attended the $A \& E$ due to a fall or a complication of a fall. Furthermore all of the 20 patients subsequently admitted presented initially with symptoms related to a fall and 18 of these lived alone. The results suggest that elderly patients who attend $A \& E$ are a high risk group, particularly those who present with a fall and are living alone. The diagnoses missed were generally not life threatening but would interfere significantly with the patient's quality of life. We suggest that $\mathrm{A} \& \mathrm{E}$ departments should have a special mechanism for referring elderly patients living alone who are to be discharged from $\mathrm{A} \& \mathrm{E}$ following a fall.

1 Currie CT, Lawson PM, Robertson CE, Jones A. Elderly patients discharged from an accident and emergency patients discharged from an accident and emergency department - their depe

2 Currie CT, Runciman P, Nicol M, Robertson CE. Health visitor follow-up of elderly patients discharged from an accident and emergency department: evaluation of effectiveness, development of screening instrument. Age Ageing 1994;23(suppl 1):6-7.

3 Williamson J, Stokoe IH, Gray S, Fisher M, Smith S, McGhee A, et al. Old people at home; their unreported needs. Lancet 1964;i:1117-20.

4 Hegarty V, Donovan MC, Pye M, Khaw KT. Screening the over 75's - what about the non-responders? Age Ageing 1994;23(suppl 1):12-13. 